\title{
Understanding the Journey of Dopant Copper lons in Atomically Flat Colloidal Nanocrystals of CdSe Nanoplatelets Using Partial Cation Exchange Reactions
}

\author{
Manoj Sharma, ${ }^{\dagger, \ddagger}$ Murat Olutas, ${ }^{\ddagger}, \S$ Aydan Yeltik, ${ }^{\ddagger}$ Yusuf Kelestemur, ${ }^{\ddagger}$ Ashma Sharma, ${ }^{\dagger,}$ Savas Delikanli, ${ }^{\dagger, \ddagger}$
} Burak Guzelturk, ${ }^{\ddagger}$ Kivanc Gungor, James R. McBride, ${ }^{\| \odot}$ and Hilmi Volkan Demir ${ }^{*}, \neq$,

${ }^{\dagger}$ LUMINOUS! Center of Excellence for Semiconductor Lighting and Displays, School of Electrical and Electronics Engineering, School of Physical and Mathematical Sciences, School of Materials Science and Engineering, Nanyang Technological University, Nanyang Avenue, 639798 Singapore

${ }^{\ddagger}$ Department of Electrical and Electronics Engineering, Department of Physics, and UNAM-Institute of Materials Science and Nanotechnology, Bilkent University, Ankara 06800, Turkey

${ }^{\S}$ Department of Physics, Abant Izzet Baysal University, Bolu 14030, Turkey

"Department of Chemistry and Vanderbilt Institute for Nanoscale Science and Engineering, Vanderbilt University, Nashville, Tennessee 37235, United States

\section{Supporting Information}

ABSTRACT: Unique electronic and optical properties of doped semiconductor nanocrystals (NCs) have widely stimulated a great deal of interest to explore new effective synthesis routes to achieve controlled doping for highly efficient materials. In this work, we show copper doping via postsynthesis partial cation exchange (CE) in atomically flat colloidal semiconductor nanoplatelets (NPLs). Here chemical reactivity of different dopant precursors, reaction kinetics, and shape of seed NPLs were extensively elaborated for successful doping and efficient emission. Dopant-induced Stokesshifted and tunable photoluminescence emission (640 to $830 \mathrm{~nm}$ ) was observed in these $\mathrm{Cu}$-doped $\mathrm{CdSe}$ NPLs using different thicknesses and heterostructures. High quantum yields (reaching $63 \%)$ accompanied by high absorption cross sections ( $>2.5$ times) were obtained in such NPLs compared to those of Cu-doped CdSe colloidal quantum dots (CQDs). Systematic tuning of the doping level in these two-dimensional NPLs provides an insightful understanding of the chemical dopant based orbital hybridization in NCs. The unique combination of doping via the partial CE method and precise control of quantum confinement in such atomically flat NPLs originating from their magic-sized vertical thickness exhibits an excellent model platform for studying photophysics of doped quantum confined systems.

\section{INTRODUCTION}

Doping of transition metal ions into semiconductor colloidal quantum dots (CQDs) enables exciting electronic and optical properties. $^{1-6}$ In the past two decades, a great deal of attention has been given to explore the new synthesis methods for the electronic doping of CQDs to understand the resulting novel properties and to use them in optoelectronic applications. $^{2-4,7-10}$ With this overarching goal, different synthesis methods have been developed for the preparation of such doped nanomaterials from the molecular precursors. ${ }^{11}$ These direct synthesis methods require careful control of nucleation, growth, and surface chemistry for achieving the desired morphology and a different range of optical to electronic properties. $^{12-15}$

On the other hand, cation exchange (CE) has emerged as a simple and versatile tool for the synthesis of different

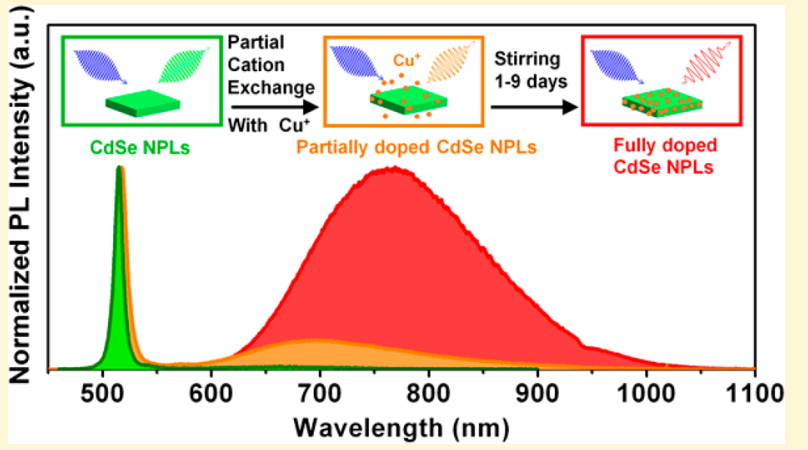

nanomaterials, which are otherwise difficult to synthesize through other conventional synthetic routes. ${ }^{16,17} \mathrm{CE}$ reactions allow for the necessary postsynthesis modifications of the presynthesized nanocrystals (NCs) into stepwise-controlled new complex nanomaterials. ${ }^{16-19}$ Accompanied by the success of the full $\mathrm{CE}$ method for obtaining new nanomaterials from existing presynthesized NC seeds, in recent years, the partial $\mathrm{CE}$ method has been a favorable alternative for the doping of semiconductor CQDs. ${ }^{2,20}$ In partial CE reactions, a few of the dopant cations are replaced by the host cation in the crystal lattice leading to interesting electronic, optical, and magnetic properties. ${ }^{2,20}$ Using the partial CE reactions at room- and

Received: January 15, 2018

Revised: $\quad$ May 3, 2018

Published: May 3, 2018 
moderately high-temperatures, successful doping has been shown for binary (e.g., $\mathrm{CdSe}, \mathrm{ZnSe})^{2,3}$ and ternary (e.g., $\mathrm{CuInS}, \mathrm{ZnInS})^{21}$ semiconductor CQDs.

In particular, the $\mathrm{Cu}(\mathrm{I})$ doping of $\mathrm{CdSe} C Q D$ s has been shown via heat-up methods and, more recently, by a hightemperature CE method. ${ }^{3,10}$ As such, successful doping at the substitutional sites has resulted in $\mathrm{Cu}(\mathrm{I})$-related emission. Increased $\mathrm{Cu}(\mathrm{I})$ ion doping in $\mathrm{CdSe}$ CQDs synthesized through heat-up methods exhibit redshifting dopant emission spectra. ${ }^{22}$ Using theoretical and experimental methods, it has been shown that orbital hybridization between the chemical dopant and the host cation $(\mathrm{Cd})$ results in a shift of the conduction band $(\mathrm{CB})$ edge only, which in turn redshifts the $\mathrm{Cu}(\mathrm{I})$ emission with the enhancement in the doping concentrations. ${ }^{22}$ However, it is difficult to control the size of NCs at different doping levels, which interferes with the orbital hybridization induced redshift in the photoluminescence (PL) emission spectra. The partial CE method allows for the use of the same core for adding different amounts of chemical dopants. Such presynthesized cores having exactly the same size distribution can serve as an excellent host for comparing the PL emission spectra of differently doped quantum confined systems. Sahu et al. previously reported on doping different amounts of silver $(\mathrm{Ag})$ in CdSe CQDs, ${ }^{2}$ and later the doping of $\mathrm{Cu}$ and $\mathrm{Ag}$ in CQDs was also reported by using a similar aforementioned partial CE reactions. ${ }^{20}$ However, in none of these studies efficient dopant induced emission was found.

Atomically flat NCs, also known as colloidal nanoplatelets (NPLs), or alternatively as "colloidal quantum wells" (CQWs), have attracted great interest in the past few years among all colloids thanks to their strong $1 \mathrm{D}$ confinement along with their magic-sized vertical thicknesses and assorted heterostructures (core-crown, core/shell, core/crown/shell)). ${ }^{23-25}$ As compared to the CQDs, this new class of NCs features superior optical properties including narrow spontaneous emission spectra, suppressed inhomogeneous emission broadening, extremely large linear and nonlinear absorption cross sections, ${ }^{26,27}$ and giant oscillator strengths. ${ }^{28,29}$ Recently, using full CE methods, core only CdSe NPLs have been shown to be converted into $\mathrm{Cu}_{2-x} \mathrm{Se}$ and $\mathrm{HgSe}$ NPLs. ${ }^{30,31}$ In addition to the core only NPLs, CdSe/CdS core/shell NPLs have been also successfully converted into $\mathrm{Cu}_{2} \mathrm{Se} / \mathrm{Cu}_{2} \mathrm{~S}, \mathrm{ZnSe} / \mathrm{ZnS}$, and $\mathrm{PbSe} / \mathrm{PbS}$ NPLs. ${ }^{19}$ However, unlike CQDs, there is no systematic study for electronic doping of these CQWs using partial CE reactions. Furthermore, the observation of pure vertical confinement in these atomically flat CQWs makes them a highly appealing model host system for studying variable doping effects without considering variation in the quantum confinement effect.

Very recently, we have shown successful $\mathrm{Cu}(\mathrm{I})$ doping in 3$4 \mathrm{ML}$ of CdSe NPLs using a high-temperature nucleation doping method. ${ }^{32}$ These doped NPLs have shown large Stokesshifted dopant-induced emission in red to near-infrared (NIR) regions of the electromagnetic spectrum with high quantum yields ( $\sim 97 \%)$. Furthermore, they were successfully shown to be applied as luminescent solar concentrators (LSCs) for harvesting solar light. However, limited control over the doping level with the nucleation doping method makes it practically impossible to understand the doping mechanism and the origin of this efficient dopant emission from these vertically magicsized NPLs (e.g., $\sim 1.2 \mathrm{~nm}$ for $4 \mathrm{ML} \mathrm{CdSe}$ ). Therefore, we hypothesized that partial $\mathrm{CE}$ reactions can serve as a convenient method enabling the precise control of the doping level and its resulting excitonic features by using precursor reactivity, time, and temperature of reactions. Furthermore, doping of $\mathrm{Cu}(\mathrm{I})$ into NPLs having different thicknesses and heterostructures (e.g., $5 \mathrm{ML} \mathrm{CdSe}$ and core/shell NPLs) could possibly be achieved with the CE method to further tune their highly desired excitonic features.

Here, we propose and develop the $\mathrm{Cu}$-doped CdSe NPLs via the postsynthesis partial CE method. In this work, we carried out a systematic and detailed study to understand the effect of doping into these NPLs with the use of slowly varying amounts of $\mathrm{Cu}(\mathrm{I})$ ions. Various techniques including inductively coupled plasma mass spectroscopy (ICP-MS), high-angle annular darkfield (HAADF) scanning transmission electron microscopy (STEM) combined with energy-dispersive X-ray spectroscopy (EDS) mapping and X-ray photoelectron spectroscopy (XPS) have been used for the confirmation of $\mathrm{Cu}(\mathrm{I})$ doping. With the doping of core-only NPLs having different thicknesses (3-5 ML), we achieved tunable dopant-induced emission covering a wide range of the electromagnetic spectrum from the visible to NIR. The combined analyses of these results from the excitation-dependent steady-state PL spectroscopy and highresolution XPS depth profile have demonstrated successful doping of $\mathrm{Cu}(\mathrm{I})$ ions in the host medium of the CdSe NPLs, and this is responsible for the dominant and strong dopantrelated tunable PL emission with reduced self-absorption and high PL quantum efficiencies reaching 63\%. By controlling kinetics of the partial $\mathrm{CE}$, we studied the journey of $\mathrm{Cu}$ ions from the interstitial to the deep substitutional sites. ${ }^{16}$ Intentional slow doping via partial CE reactions has allowed us to understand chemical dopant induced orbital hybridization of the host cadmium (Cd) ions. Furthermore, the underlying mechanism of this efficient dopant-induced PL emission has been investigated and understood by using temperaturedependent time-resolved fluorescence (TRF) spectroscopy.

\section{RESULTS AND ANALYSES}

Using the partial CE method, the atomically flat CdSe NPLs having different vertical thicknesses (e.g., 3-5 MLs) have been doped with $\mathrm{Cu}(\mathrm{I})$ ions (Figure 1a). First, we synthesized the 4 ML thick undoped core-only CdSe NPLs having a zinc-blende crystal structure with an earlier reported recipe. ${ }^{23,24}$ These

(a)
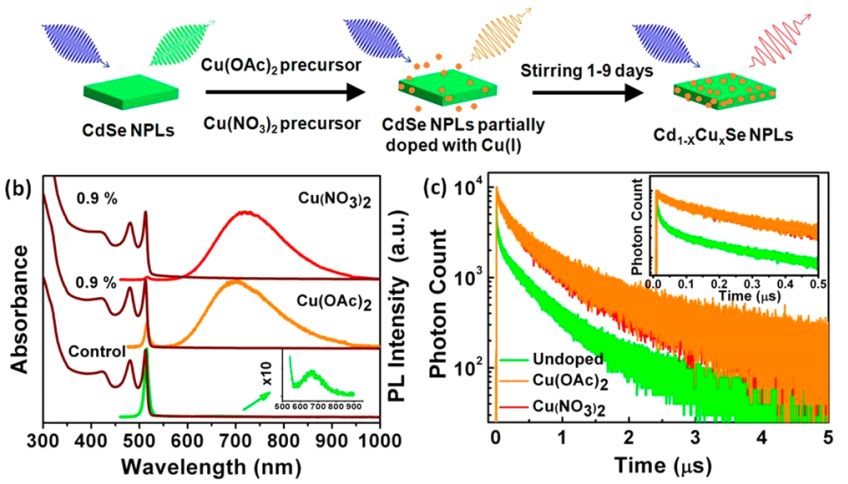

Figure 1. (a) Copper doping through partial cation exchange reaction by using copper-acetate and copper-nitrate precursors. (b) UV-visible absorption and normalized photoluminescence spectra and (c) roomtemperature time-resolved fluorescence decay curves for undoped and 0.9\% Cu-doped $4 \mathrm{ML}$ CdSe NPLs synthesized using copper-acetate and copper-nitrate precursors. Inset in (b) shows the 10 times magnified view of weak defect emission for the case of undoped NPLs. 

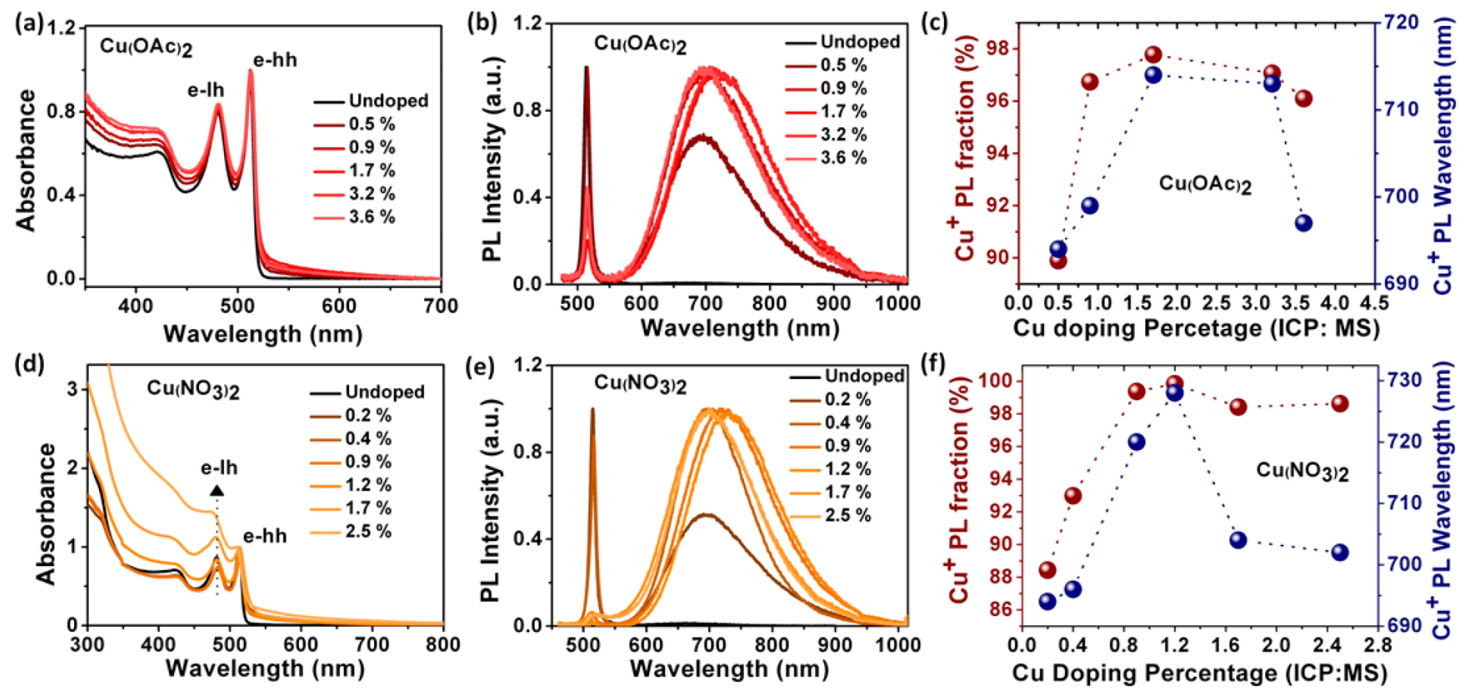

Figure 2. UV-visible absorption spectra and steady-state photoluminescence emission spectra of Cu(I)-doped CdSe NPLs (a, b) using copper(II) acetate, $(\mathrm{d}, \mathrm{e})$ using copper(II) nitrate trihydrate precursors, and (c, f) variation of $I_{\text {dopant }} / I_{\text {total }}$ emission $\left(\mathrm{Cu}^{+} \mathrm{PL}\right.$ fraction) and peak emission wavelength with increasing $\mathrm{Cu}(\mathrm{I})$ to $\mathrm{Cd}(\mathrm{II})$ values for both series synthesized by acetate and nitrate precursors of copper, respectively.

NPLs were used for the synthesis of Cu-doped CdSe NPLs with varying $\mathrm{Cu}(\mathrm{I})$ concentrations. It has been recently reported that CdSe NPLs can be rapidly converted into $\mathrm{Cu}_{2-x}$ Se NPLs because the $d$-spacing of bulk CdSe and bulk $\mathrm{Cu}_{2-x} \mathrm{Se}$ differ by only 5-6\%, which enhances the $\mathrm{CE}$ with $\mathrm{Cu}(\mathrm{I}){ }^{16,30}$ In our study, to avoid the full $\mathrm{CE}$, we used trioctylphosphine (TOP) as the surfactant to controllably mediate the incorporation of $\mathrm{Cu}(\mathrm{I})$ ions into the CdSe NPLs as the dopant (see the methods section for the detailed information). Briefly, variable amounts of ethanolic solutions of $\mathrm{Cu}(\mathrm{OAc})_{2}$ and $\mathrm{Cu}\left(\mathrm{NO}_{3}\right)_{2}$ premixed with TOP were added to the known concentrations of undoped $4 \mathrm{ML}$ CdSe NPLs under stirring in a nitrogen-filled glovebox at $50^{\circ} \mathrm{C}$ (Figure 1a). The partial CE reaction was completed after $1 \mathrm{~h}$ with the addition of ethanol and repeated washing of the NPL samples. Finally, all the samples were dispersed in toluene and kept on stirring for $1-9$ days at $50{ }^{\circ} \mathrm{C}$ under ambient conditions. Various optical measurements were performed every day to understand the evolution of dopant emission and reactivity of different dopant precursors.

Figure $1 \mathrm{~b}$ depicts the UV-visible absorption and steady-state $\mathrm{PL}$ spectroscopy results for the undoped and $0.9 \% \mathrm{Cu}$-doped 4 ML CdSe NPLs (as an exemplary case). In the absorption spectrum, both electron-heavy hole (e-hh) and electron-light hole (e-lh) transitions for the doped NPLs stay unchanged, suggesting that $0.9 \% \mathrm{Cu}(\mathrm{I})$ doping does not alter the discrete excitonic features of the NPLs. The normalized PL emission spectrum for the undoped CdSe NPL exhibits a spectrally narrow emission peak at $\sim 513 \mathrm{~nm}$ having a full-width at halfmaximum (fwhm) of $\sim 10 \mathrm{~nm}$, which is characteristic to the CdSe NPLs having $4 \mathrm{ML}$ vertical thickness. ${ }^{24}$ For the dopedNPLs, a large Stokes-shifted broad emission at a higher wavelength region $(>650 \mathrm{~nm})$ is observed in addition to the band-edge ( $\mathrm{BE}$ ) emission at $\sim 513 \mathrm{~nm}$. Herein, origin of the broad and Stokes-shifted emission appears to be similar to $\mathrm{Cu}(\mathrm{I})$ doped CdSe CQDs. ${ }^{33,34}$ Briefly, this kind of broadband emission has been observed to originate from the recombination of delocalized electrons in the $\mathrm{CB}$ with strongly localized holes in $\mathrm{Cu}(\mathrm{I})$ states and is referred to as metal-to-ligand (conduction-band) charge transfer $\left(\mathrm{ML}_{\mathrm{CB}} \mathrm{CT}\right)$ emission.
Therefore, the midbandgap position of $\mathrm{Cu}(\mathrm{I})$ dopant ions results in the Stokes-shifted emission.

To deeply understand the emission profiles of all these doped and undoped NPLs, time-resolved fluorescence spectroscopy (TRF) using a time-correlated single photon counting system (TCSPC) was utilized. In the undoped NPL ensemble, a broad defect emission was observed (Figure 1b). The fluorescence decay curve of this defect emission is shown in Figure 1c, in which the decay was also compared to that of the dopant-related emission in the doped NPL ensembles. There is a stark contrast between the decay curves, which indicates that the large Stokes-shifted emission in the doped ensemble is not due to the surface trap states but originates from the excited state between the CB of the CdSe NPL and the localized hole state of the $\mathrm{Cu}$ dopant. This observation is consistent with the previous literature on the Cu-doped NCs. ${ }^{1,34-38}$ Average lifetimes for the dopant emission are 512 and $480 \mathrm{~ns}$ for acetate and nitrate precursor based samples, respectively, whereas the average lifetime of defect emission from the undoped NPLs is 180 ns. Indeed, the PL lifetime of the transition metal ions such as $\mathrm{Mn}$ and $\mathrm{Cu}$ is very long as stated in the previous reports for CQDs. ${ }^{4,34,37,39}$ This implies that the $\mathrm{Cu}(\mathrm{I})$-related emission in NPLs appears similar in nature with 3D confined CQDs.

For the systematic investigation of this large Stokes-shifted emission in the doped NPLs and the variation in the contribution of $\mathrm{BE}$ to the dopant-related emissions, we synthesized a wide range of $\mathrm{Cu}(\mathrm{I})$-doped CdSe NPLs by increasing both acetate and nitrate precursors in the partial CE reactions. Copper to cadmium ratios (i.e., $\mathrm{Cu}(\mathrm{I}): \mathrm{Cd}(\mathrm{II})$ ) were obtained for all the samples via ICP:MS measurements. To this end, all the doped samples were washed extensively with ethanol before the ICP measurements to remove any unreacted $\mathrm{Cu}(\mathrm{I})$ ions or excess $\mathrm{Cd}$ ions on the NPL surface. Figures 2(ac) and $2(\mathrm{~d}-\mathrm{f})$ depict the results and their analyses from the UV-visible absorption and steady-state PL spectroscopies for the undoped and $\mathrm{Cu}(\mathrm{I})$-doped $4 \mathrm{ML}$ CdSe NPLs using acetate and nitrate based copper precursors, respectively. Absorption spectra for all samples using acetate precursors suggest e-hh and e-lh transitions remained unaffected at all studied doping levels. 

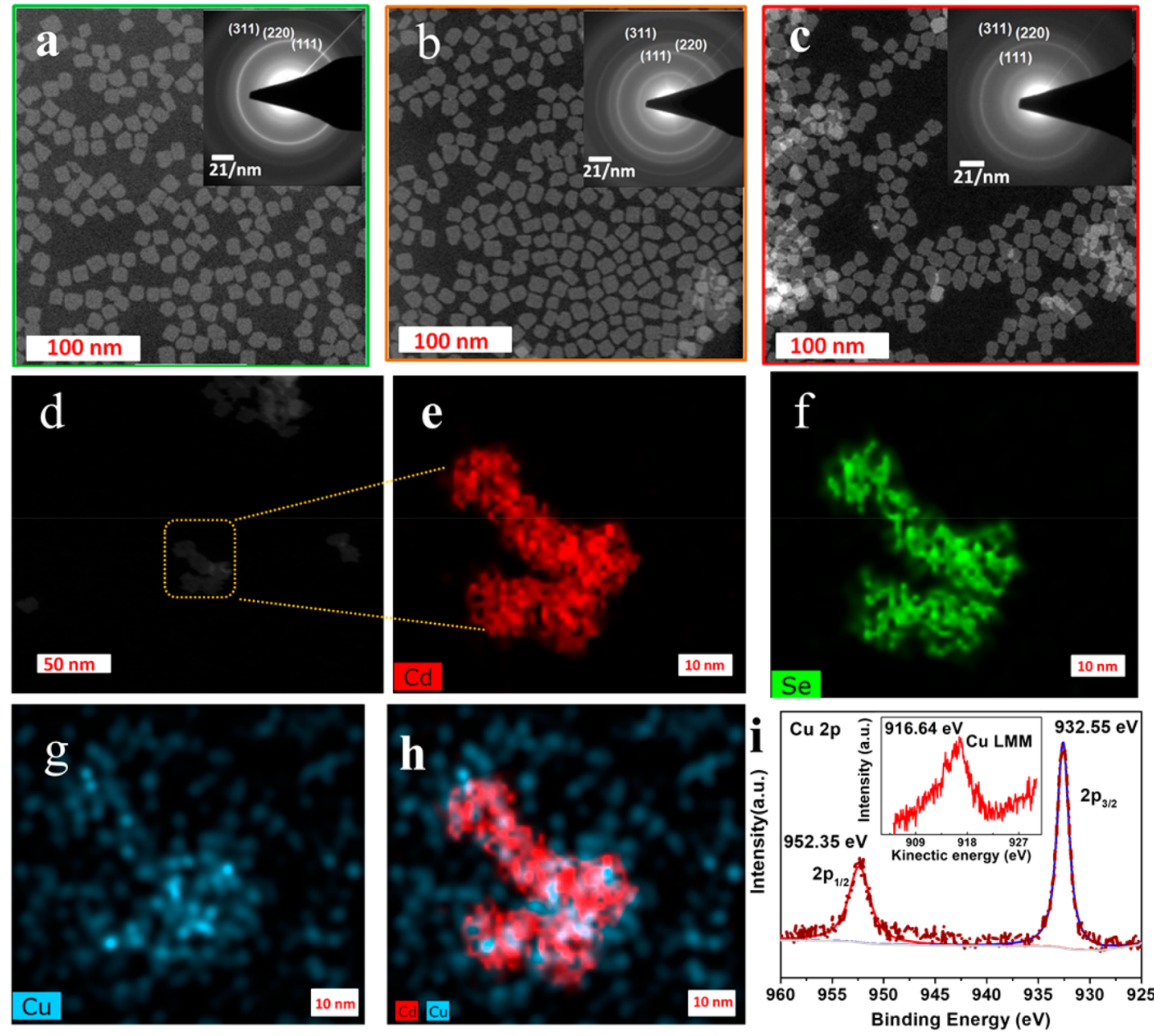

Figure 3. (a, b) HAADF-TEM images of (a) undoped and (b, c) $0.9 \%$ of Cu-doped NPLs using acetate and nitrate precursors. Insets of parts a and c show the SAED pattern for the corresponding samples, (d) HAADF-STEM images of 3.6\% of Cu-doped 4 ML CdSe NPLs, (e-g) EDS maps of cadmium, selenium, and copper, (h) overlaid EDS map of cadmium and copper, and (i) high-resolution XPS spectra of Cu (2p) orbitals. The inset of part (i) shows Auger spectra of Cu LMM orbitals.

On the other hand, consistent increase in the nitrate precursors leads to degradation of excitonic features of the NPLs (see Figure $2 \mathrm{~d}$ and Figure $\mathrm{S} 1$ ). In the absorption spectra for the doped samples, there exists a weak tail along with e-hh transition (at the lower photon energy) as compared to the undoped NPLs (Figures $2 \mathrm{a}$ and $2 \mathrm{~d}$ ), which is attributed to the metal-to-ligand (conduction band) charge transfer $\left(\mathrm{ML}_{\mathrm{CB}} \mathrm{CT}\right.$ ) absorption state for the Cu-doped CdSe CQDs. ${ }^{3,36}$

Figures $2 \mathrm{~b}$ and $2 \mathrm{e}$ show the $\mathrm{PL}$ emission spectra of $\mathrm{Cu}(0-$ $3.6 \%)$ and $\mathrm{Cu}(0-2.2 \%)$ doped NPLs with the use of copperacetate and -nitrate precursors, respectively. For both cases, an increase in the $\mathrm{Cu}$-doping results in an increase in the dopantrelated Stokes-shift of the emission with respect to the $\mathrm{BE}$ emission. However, the variation in relative intensities between the $\mathrm{BE}$ and dopant-related emissions as a function of the $\mathrm{Cu}(\mathrm{I})$ concentration in the doped NPLs seems more complex than similarly doped CdSe CQDs studied in the related literature. ${ }^{3,34,35}$ To follow these variations, we summarized useful information from the emission spectra for both series given in Figures $2 \mathrm{c}$ and $2 \mathrm{f}$. Concisely, this summary illustrates that the fraction of total integrated PL intensity associated with the $\mathrm{ML}_{\mathrm{CB}} \mathrm{CT}$ emission ( $I_{\text {dopant }} / I_{\text {tot }}$ ), and its peak emission wavelength changes with the increase of the $\mathrm{Cu}(\mathrm{I}): \mathrm{Cd}(\mathrm{II})$ ratio in the CdSe NPL host medium. In Figure $2 c$, the emission contribution coming from dopant ions $\left(\mathrm{Cu}^{+} \mathrm{PL}\right.$ fraction) becomes monotonically stronger as the $\mathrm{Cu}^{+}$concentration is increased up to the value of $1.7 \%$. For $\mathrm{Cu}(\mathrm{I})$ concentration of $1.7 \%$, the dopant emission has the highest overall contribution (97.8\%). For the higher $\mathrm{Cu}(\mathrm{I})$ concentrations above $1.7 \%$, the $\mathrm{BE}$ emission starts to recover again, and, hence, the dopant emission contribution starts to decrease. Interestingly, the PL peak emission wavelength for the dopant-related emission also redshifts in a monotonic manner with the increasing dopant concentrations up to $1.7 \%$. Thereafter, it follows dopant emission contributions and starts to blueshift.

We also performed PL excitation (PLE) measurements to find out the origin of the large Stokes-shifted PL emission in the doped NPLs. Figure S2 shows the excitation spectra of the $\mathrm{Cu}$-doped NPLs with varying $\mathrm{Cu}$ concentrations $(0.9,1.7$, and $3.2 \%)$. The excitation spectrum of the large Stokes-shifted PL emission essentially resembles the absorption spectrum of the doped NPLs (see Figure 2a), which indicates that the large Stokes-shifted emission originates from the doped NPLs. Further, the PL excitation spectra measured for different spectral positions of the longer-wavelength emission peak (i.e., at the peak, red- and blue-tails) do not show any discernible spectral difference (see Figure S3). It suggests that there is no 

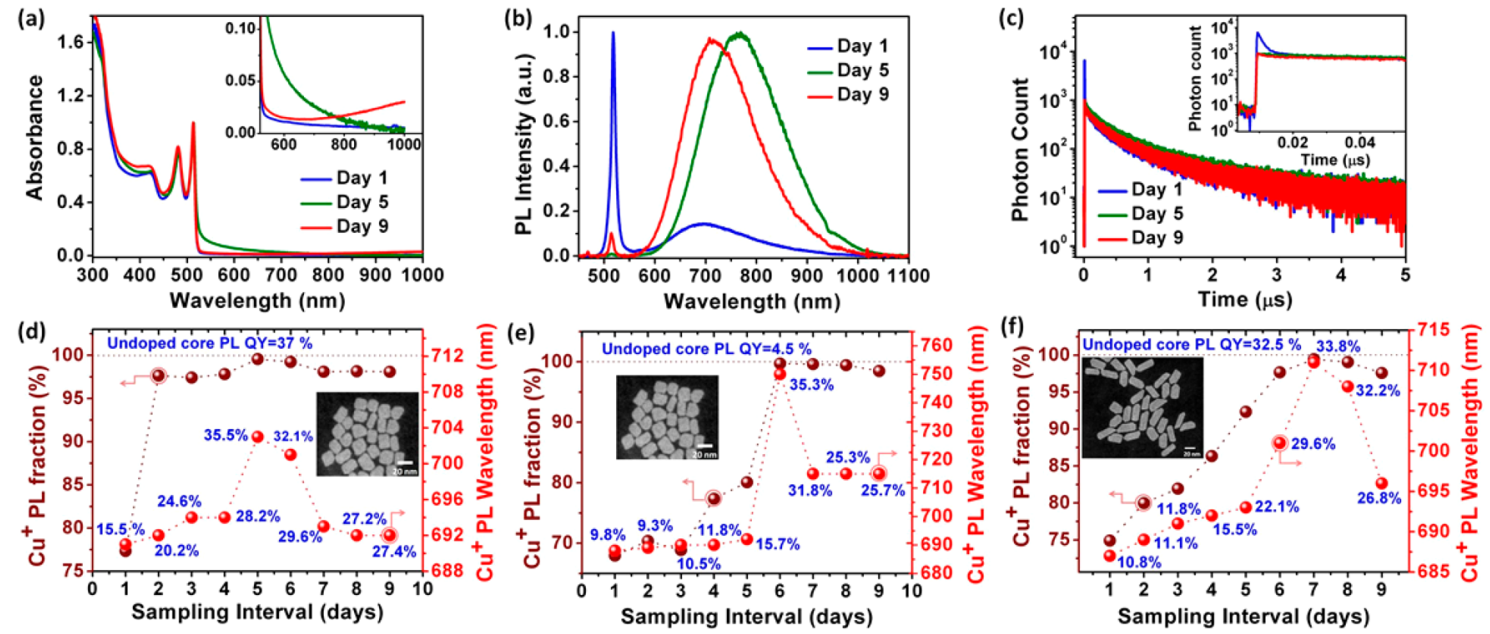

Figure 4. Mechanism of doping: (a-c) UV-visible absorption, steady-state emission, and TRF decays for Cu-doped CdSe NPLs on different days of partial CE synthesis and $(\mathrm{d}-\mathrm{f})$ variation of $I_{\text {dopant }} / I_{\text {total }}$ emission $\left(\mathrm{Cu}^{+}\right.$PL fraction) and peak emission wavelength with time for three different samples doped under identical conditions. Lateral area of samples in (d) and (e) is the same with each other i.e. $170.3 \mathrm{~nm}^{2}$; however, the initial PL QYs of their core NPLs are different. Lateral area of NPLs given in (f) is $366.6 \mathrm{~nm}^{2}$. Scale bars in all inset TEM micrographs are $20 \mathrm{~nm}$.

inhomogeneous broadening in the doped NPLs thanks to their magic-sized vertical thicknesses.

In the case of doping with nitrate precursors, almost complete dopant emission appears at the $1.2 \% \mathrm{Cu}(\mathrm{I})$ doping levels (Figures $2 \mathrm{e}$ and $2 \mathrm{f}$ ). Thereafter, a further increase in doping percentage results in a slight decrease of dopant contribution in the PL emission spectrum. Consistently, the PL peak emission wavelength in this case also follows the same trend. Here, contrary to the cases of acetate precursor shown in Figure $2 \mathrm{~b}$, almost full dopant emission emerges at $1.2 \%$ of $\mathrm{Cu}(\mathrm{I})$ doping. This suggests that the use of different precursors affect the emission dynamics. The different reactivity of both precursors might affect distribution and nature (interstitial/ substitutional) of the doping in these atomically flat NPLs.

Contribution of the $\mathrm{BE}$ emission in the doped NPL ensembles can be explained by two different hypotheses. The first is that all of the NPLs are doped and the BE emission appears from the doped NPLs, which has been shown for the $\mathrm{Cu}(\mathrm{II})$ doping in the NCs. ${ }^{6}$ This previous report suggests $\mathrm{Cu}$ existing in +2 oxidation state creates "persisting holes", which can allow for the $\mathrm{BE}$ recombination competing with $\mathrm{Cu}(\mathrm{II})$ related recombination. However, most of the research suggests the second hypothesis that $\mathrm{Cu}$ is doped as the +1 oxidation in CdSe CQDs, upon photoexcitation which converts to the +2 state, resulting in a dominant Stokes-shifted dopant emission. $3,33,40,41$ Therefore, $\mathrm{Cu}$ having the +1 oxidation state in CdSe CQDs cannot fundamentally allow any BE emission in the $\mathrm{Cu}(\mathrm{I})$-doped NCs. The only possibility for coappearance of the $\mathrm{BE}$ and dopant emissions can result from two different subpopulations within the doped ensemble: doped and undoped NCs. This second hypothesis has been recently verified via single-particle measurements for $0.6 \% \mathrm{Cu}(\mathrm{I})$-doped CdSe CQDs. ${ }^{34}$ However, in our case, apart from the undoped subpopulation, uneven distribution of dopant ions among individual NPLs may also result in the BE emission. Since, the lateral dimension of these 4 ML NPLs $(14.0 \pm 1.5 \mathrm{~nm} \times 12.9 \pm$ $1.9 \mathrm{~nm}$ ) is large in comparison with previously studied $\mathrm{Cu}(\mathrm{I})$ : CdSe CQDs $(\sim 3.5 \mathrm{~nm})$. Moreover, due to the different reactivity of the acetate and nitrate precursors during partial $\mathrm{CE}$ reactions, there is a possibility of different doping distribution among individual NPLs. As seen from Figures $2 \mathrm{c}$ and $2 \mathrm{f}$, at similar doping levels (0.9\%), nitrate precursor based doped NPLs show nearly full dopant emission contribution (99.4\%) as compared to acetate precursor based doped NPLs (96.7\%). This suggests nitrate precursor reacts faster in the CE reactions as compared to acetate ones, or in other terms they provide more uniform distribution of $\mathrm{Cu}(\mathrm{I})$ dopant ions among $\mathrm{CdSe}$ NPLs. Furthermore, the appearance of BE emission at higher doping values (e.g., $>1.7 \%$, see Figure $2 b$ ) decreases the probability of subpopulations. Therefore, further investigations are needed for understanding the emission mechanism for these newly studied $\mathrm{Cu}$-doped NPLs.

Structural and elemental investigations were also conducted to verify the $\mathrm{Cu}(\mathrm{I})$ doping and its distribution among the NPLs studied in this work. High-angle annular dark-field transmission electron microscopy (HAADF-TEM) images of the undoped and $\mathrm{Cu}$-doped CdSe NPLs (for the exemplary cases of 1.7 and $1.2 \% \mathrm{Cu}(\mathrm{I})$-doped samples from acetate- and nitrate-based samples) present regular rectangular shapes with average lateral dimensions of $14.0 \pm 1.5 \mathrm{~nm}$ by $12.9 \pm 1.9 \mathrm{~nm}$ and a vertical thickness of $1.2 \mathrm{~nm}$ corresponding to $4 \mathrm{ML}$ of CdSe (Figures $3 a-3 c)$. Here it was observed that there is no significant change in the lateral size distribution of NPLs (see also Figures S4a and $\mathrm{S} 4 \mathrm{~b})$ and their crystallinity after the $\mathrm{Cu}$-doping process (compared to high-resolution bright-field TEM images of the undoped, $1.7 \% \mathrm{Cu}(\mathrm{I})$-doped NPLs in Figures S5a and S5b). Figure S6 exhibits the XRD pattern acquired from the undoped and doped NPLs and shows their characteristic peaks arising from the zinc-blende structure. In the doped samples, structural features do not change as compared to the undoped samples, which has been also observed in the selected area electron diffraction (SAED) pattern as shown in the inset of Figures $3 a-3 c$.

The presence of copper ions in the host CdSe NPLs has been confirmed through HAADF-STEM microscopy with individual energy dispersive spectroscopy (EDS) maps for cadmium $(\mathrm{Cd})$, selenium $(\mathrm{Se})$, and copper $(\mathrm{Cu})$. Figure $3 \mathrm{~d}$ shows the HAADF image of $3.6 \% \mathrm{Cu}$-doped NPLs. For the selected group of NPLs shown in Figure $3 \mathrm{~d}$, individual elemental maps are presented in Figures $3 \mathrm{e}-3 \mathrm{~g}$. Figure $3 \mathrm{~h}$ shows the overlaid EDS map of $\mathrm{Cd}$ and $\mathrm{Cu}$. Even at very low doping levels, we can explicitly observe the presence of $\mathrm{Cu}$ ions 
in the NPLs along with Cd and Se. However, considering the instrumental limits the identification of the exact location and distribution of $\mathrm{Cu}$ dopant ions in NPLs is practically not possible. At the same time, the HAAD-STEM microscopy with individual EDS maps along with EDS spectra still provide useful information by confirming successful $\mathrm{Cu}$ doping in CdSe NPLs (Figures $3 \mathrm{e}-3 \mathrm{~g}$ and Figures $\mathrm{S} 7-\mathrm{S} 11$ ).

To further verify the $\mathrm{Cu}$-doping in the NPLs, we employed XPS measurements, the results of which also prove the successful doping in the NPLs. Apart from showing the presence of $\mathrm{Cu}$ in the NPLs, XPS additionally provides insight about the possible oxidation states for these dopant ions. For the analysis, all of the peaks have been spectrally corrected according to carbon 1s standard peak. Figure $3 \mathrm{i}$ shows the highresolution XPS spectra of 3.6\% Cu-doped NPLs for $\mathrm{Cu} 2 \mathrm{p}$ orbitals. We find $\mathrm{Cu} 2$ p-peaks at 932.55 and $955.35 \mathrm{eV}$ corresponding to $2 \mathrm{p}_{1 / 2}$ and $2 \mathrm{p}_{3 / 2}$ orbitals, respectively. ${ }^{30,37,42}$ Spin-orbital splitting of $\mathrm{Cu}(\mathrm{I})$ ions in our case is $19.8 \mathrm{eV}$, which is in good agreement with the reported value of $19.6 \mathrm{eV}$ in the literature. ${ }^{10,37}$ Moreover, the absence of any satellite peaks strongly suggests the absence of $\mathrm{Cu}$ (II) in our doped NPLs. ${ }^{37,42}$ Therefore, these well screened peaks in our spectra indicate the presence of either $\mathrm{Cu}(\mathrm{I})$ or $\mathrm{Cu}(0)$ oxidation state in the CdSe NPLs. The Auger Cu LMM spectrum further offers that the valence state of $\mathrm{Cu}$ in the NPLs should be $1+$ (see the inset of Figure 3i), which is in accordance with the recent literature of $\mathrm{Cu}(\mathrm{I})$ doped CdSe CQDs. ${ }^{16,22,36}$ Additional XPS analysis, survey-spectrum, and high-resolution XPS for $\mathrm{Cd}$ and Se are provided in Figures S12 and S13.

Different elemental characterizations apparently evidence the presence of $\mathrm{Cu}(\mathrm{I})$ dopant ions in the host CdSe NPLs. However, it is difficult to see the doping distribution among these atomically flat individual NPLs. Therefore, to understand doping distribution and $\mathrm{BE}$ to dopant-related emission contribution among individual NPLs, we carried out additional experiments by carefully varying the reaction time of the partial CE process, doping concentration, and initial PL quantum yield of the cores and by using different lateral sized control groups of undoped NPLs. Detailed optical characterizations were conducted every day to understand evolution of dopant-related $\mathrm{PL}$ emission and the emission dynamics (Figures $4 \mathrm{a}-4 \mathrm{f}$ and Figures S14-S17). It is worth mentioning that, to compare the dopant-related emission among different $4 \mathrm{ML}$ samples, we performed these partial $\mathrm{CE}$ reactions under exactly identical conditions. For example, all undoped $4 \mathrm{ML}$ cores possess exactly the same optical density, and their partial $\mathrm{CE}$ reactions were followed by addition of the same amount of $\mathrm{Cu}(\mathrm{OAc})_{2}$ precursors. Therefore, results presented in Figure 4 can be compared among themselves. However, additional analyses with the use of $\mathrm{Cu}\left(\mathrm{NO}_{3}\right)_{2}$ precursors are also provided in the Supporting Information (Figure S17). Furthermore, in order to understand the kinetics of these partial $\mathrm{CE}$ reactions during first $1 \mathrm{~h}$ we measured steady-state PL emission at different time periods (Figure S18). PL emission spectra show very little difference in the dopant emission contribution during the first 1 $\mathrm{h}$ of the reaction.

Figures $4 a-4 c$ present the UV-visible absorption, PL emission, and PL decay curves of the Cu-doped NPLs exposed to stir for 1, 5, and 9 days after the partial CE reactions. Detailed analysis for each day is given in Figure 4d. As shown in Figure $4 \mathrm{a}$, on day 1 , immediately after completion of the partial $\mathrm{CE}$, all excitonic absorption features remain intact. In the PL emission spectra (shown by the blue solid line in Figure $4 b$ ), dopant-related Stokes-shifted emission can be seen along with the dominant $\mathrm{BE}$ emission. However, the $\mathrm{PL}$ emission contribution of this dopant emission with respect to the total emission is weak. As discussed above, these doped samples were kept under stirring continuously. With the passage of time during stirring, the dopant emission starts to appear. Particularly, for the day 5 measurements, the emergence of almost complete dopant emission could be observed with slight redshift in its peak wavelength. However, recovery of some BE emission with slight blueshift of this dopant emission was observed on day 9 (shown by red solid line in Figure 4b). Moreover, a slight increase in the absorption spectra (at $>800$ $\mathrm{nm}$ ) was found for the samples measured on day 9 (see the inset of Figure 4a). The PL decay curves for this dopant emission peak on day 1, 5, and 9 are shown in Figure 4c. The presence of the fast decay component along with a long dopant-related one is clearly seen from the inset of Figure 4c. With the passage of time, for the fifth day measurement, the complete elimination of this fast trap-related PL decay component was observed. These TRF measurements explicitly support the evolution of the dopant-related emission dominating over time. The evolution of $\mathrm{Cu}(\mathrm{I})$ emission fraction, change in the emission peak wavelength, and $\mathrm{QY}$ values are summed up in Figure 4d.

These results point out that, with time, the doped $\mathrm{Cu}(\mathrm{I})$ ions in the CdSe lattice, which may be located on the surface or interstitial positions, get incorporated into deep substitutional positions. $\mathrm{Cu}(\mathrm{I})$ doping into $\mathrm{CdSe} C \mathrm{CQDs}$ has been described in the literature by a two-step process starting from interstitial to deep substitutional doping. ${ }^{10,16,22}$ Therefore, intentional slowing of the partial CE doping by using a less reactive acetatebased precursor may allow for our understanding of the evolution of this Stokes-shifted emission. As shown in Figure $4 \mathrm{~d}$ with the passage of time, both dopant emission based PL fraction and QY of the sample increases which supports the transfer of the interstitial/surface present $\mathrm{Cu}(\mathrm{I})$ ions into the deep substitutional sites. Furthermore, the redshift in the $\mathrm{Cu}(\mathrm{I})$ emission peak is also synchronous with the trends of dopant emission fraction contribution and QY results. Recently, similar redshift in the $\mathrm{PL}$ emission spectra for $\mathrm{Cu}(\mathrm{I})$ doped $\mathrm{CdSe}$ CQDs has been shown with the increase in substitutional doping of $\mathrm{Cu}$ ions. ${ }^{22}$ Their theoretical and experimental studies show the increase in $\mathrm{Cu}(\mathrm{I})$ doping results in orbital hybridization of lower $\mathrm{CB}$ states, which leads to redshift in the PL emission spectra. The slow increase in $\mathrm{Cu}$ emission for acetate based samples with the prolonged time of stirring after the completion of partial CE can also be possibly explained with the increase in $\mathrm{Cu}$ doping amounts in NPLs by unreacted $\mathrm{Cu}$ ions present in the solution or surface of NPLs. In order to understand this possibility, after the completion of partial CE for $1 \mathrm{~h}$ in the glovebox, we divided the whole sample into two parts and cleaned them 2 times and 5 times respectively with ethanol. Thereafter, they were allowed to stir under ambient at $50{ }^{\circ} \mathrm{C}$ continuously for $1-10$ days. As shown in PL spectra on the final day the $\mathrm{Cu}$ PL fraction appears to be nearly similar in both of the cases (Figure S19). On purpose we normalized both of the PL spectra at their band-edge peaks. Therefore, we can compare the emergence of $\mathrm{Cu}$ PL emission for both the cases. We believe that after 5 cleanings any unreacted $\mathrm{Cu}$ precursor present in the solution or surface should be cleaned, and only doped NPLs should precipitate. However, both 2- and 5 -cleaned samples show almost similar $\mathrm{Cu}$ emission contribution. Furthermore, in both the cases, the peak position of $\mathrm{Cu}$ 

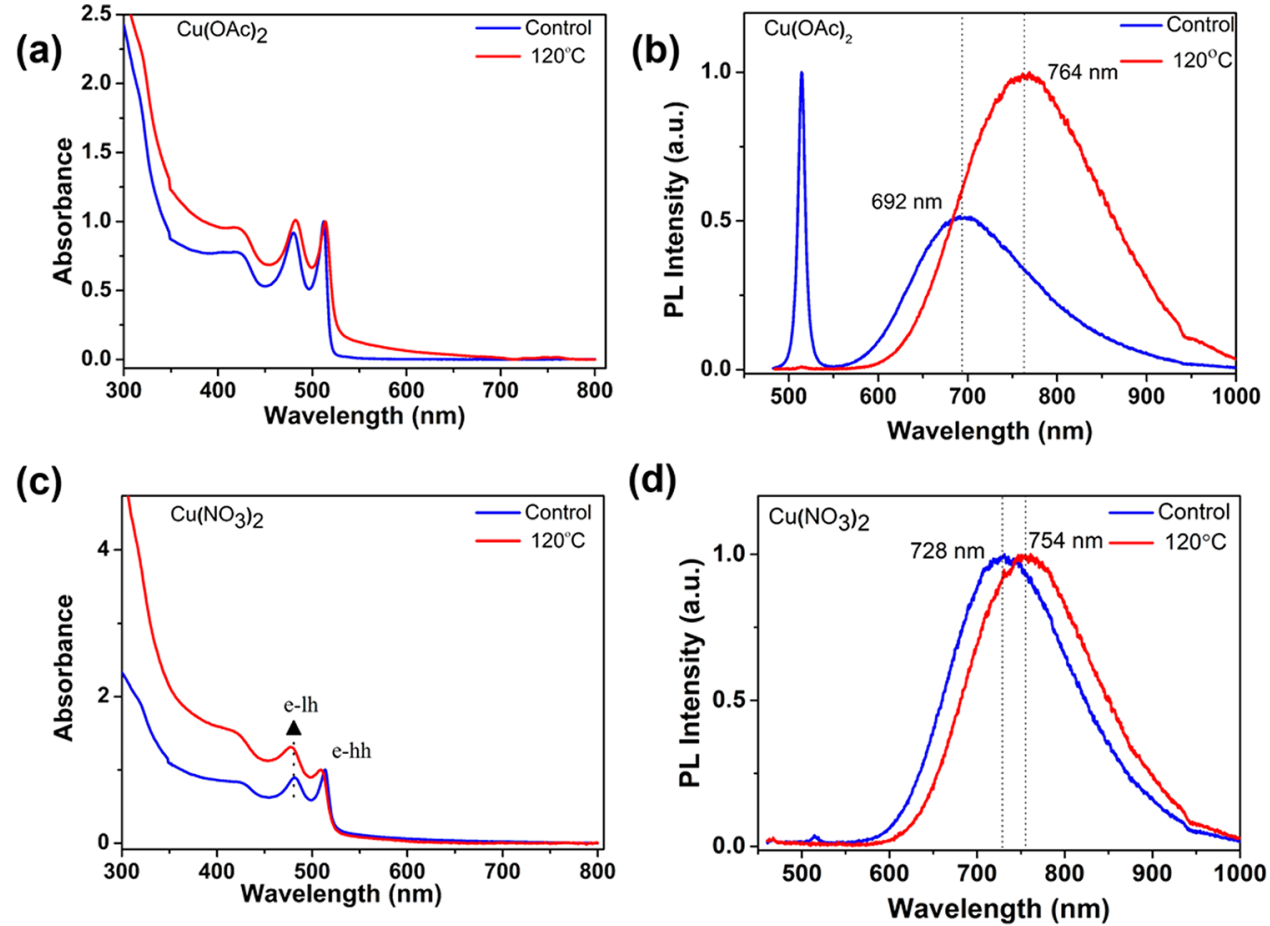

Figure 5. $(\mathrm{a}-\mathrm{d}) \mathrm{UV}$-visible absorption and PL emission spectra of $\mathrm{Cu}$-doped CdSe NPLs annealed at $120^{\circ} \mathrm{C}$ for 30 min in a nitrogen filled glovebox.

emission is also similar, which suggests the same degree of hybridization or nearly similar $\mathrm{Cu}$ doping percentage for both the cases. This result indirectly suggests that it is less likely for any unreacted $\mathrm{Cu}$ to be present in the solution. Therefore, slow rise in the dopant emission for the case of copper acetate precursor is attributed to the slow diffusion of surface/ interstitial dopant ions into substitutional sites, which result in an efficient dopant emission. Therefore, our detailed optical studies show possible deep substitution of the doping $\mathrm{Cu}(\mathrm{I})$ ions in CdSe NPLs.

As seen in Figure 4d, initial PL QY of the undoped core-only NPLs having $170.3 \mathrm{~nm}^{2}$ lateral area was measured as $32.0 \%$, whereas the highest PL QY achieved for the same NPL ensembles with the given amount of added $\mathrm{Cu}$ precursor is $33.8 \%$. In order to comprehend the role of initial PL QY of undoped core-only NPLs for the partial CE process, we cleaned the same undoped-core four times with ethanol to remove surface ligands. As expected, the removal of ligands results in a significant decrease in the PL QY of the core-only undoped NPLs. Therefore, partial CE reactions with the same sample possessing $4.5 \%$ initial $\mathrm{QY}$ were conducted under identical conditions. Figure $4 \mathrm{e}$ depicts the steady-state PL emission measurements for these experiments. Interestingly, the highest PL QY for this sample was found to be $35.3 \%$ which is very similar to the sample shown in Figure 4d. However, this poor initial PL QY sample takes 6 days to reach full $\mathrm{Cu}(\mathrm{I}) \mathrm{PL}$ fraction and the highest PL QY (Figure 4e). Briefly, the partial $\mathrm{CE}$ on both samples shows that initial QY of the undoped cores does not affect the final quality of doped samples. Therefore, only successful $\mathrm{Cu}(\mathrm{I})$ doping results in high $\mathrm{QY}$ for these doped samples.

Next, we studied $4 \mathrm{ML}$ undoped core NPLs having a large lateral area (i.e., $366.6 \mathrm{~nm}^{2}$ ) under identical experimental conditions. Figure $4 \mathrm{f}$ shows the evolution of the $\mathrm{Cu}(\mathrm{I}) \mathrm{PL}$ fraction with time and the variation of its peak wavelength along with the corresponding PL QY measurement on each day. The results indicate that the same amounts of $\mathrm{Cu}(\mathrm{I})$ dopant addition lead to almost the same final PL QY of the doped samples. However, the large lateral area of such undoped cores delays the appearance of complete dopant emission and hence achieving the highest PL QY. Clearly, the controlled emergence of dopant emission in these large starting cores as such with this partial CE method allows for the understanding of the doping mechanism in these strongly quantum confined NCs. The detailed UV-visible absorption, steady-state PL emission spectrum, and PL QY values each day for all the samples discussed in Figures $4 \mathrm{~d}-4 \mathrm{f}$ are presented in the Supporting Information (Figures S14-S16).

The recovery of $\mathrm{BE}$ emission with the delayed stirring $(\geq 7$ days), decreased PL QYs, and consistent blueshifting of the dopant-related emission peak collectively suggest the overall decrease of $\mathrm{Cu}(\mathrm{I})$ doping at the substitutional sites (see Figures $4 \mathrm{~b}$ and $4 \mathrm{~d}-\mathrm{f}$ ). At the same time, conversion of $\mathrm{Cu}$-doped $\mathrm{CdSe}$ to $\mathrm{Cu}_{2} \mathrm{Se}$ (fully or partially) may result in this blueshifting of PL emission spectrum along with the recovery of $\mathrm{BE}$ emission and overall decrease in the PL QYs. Moreover, a slight increase in absorption (at $>800 \mathrm{~nm}$ ) was also observed for almost all samples from day 7 onward (Figures S14-S16). Similar observation for the appearance of $\mathrm{Cu}_{2} \mathrm{Se}$-related absorption by conversion of $\mathrm{Cu}$-doped $\mathrm{CdSe}$ to $\mathrm{Cu}_{2} \mathrm{Se}$ has been previously reported by Gamelin et al. for CQDs. ${ }^{3}$ However, these partial $\mathrm{CE}$ reactions can be stopped at any desired point and hence preserve the desired PL emission spectra. These samples were found to be stable for over one year under ambient conditions. Earlier, as compared to CdSe CQDs, Au decorated CdSe NPLs and $\mathrm{CdSe}$ to $\mathrm{Cu}_{2-x} \mathrm{Se}$ converted NPLs were shown to be resistant to oxidation..$^{30,43,44}$ In the literature, formation of oxides on these CdSe surface is studied by Se $3 \mathrm{~d}$ core level high-resolution XPS spectra where the $\mathrm{SeO}_{2}$ peak at $58.8 \mathrm{eV}$ clearly shows the oxidation of CdSe NCs. Furthermore, Cd 3d 
spectra is also used to indicate the possible oxidation of NCs. ${ }^{44}$ They observed broadening of $\mathrm{Cd} 3 \mathrm{~d}$ after oxidation of CdSe NCs. In our case for different samples we did not observe any $\mathrm{SeO}_{2}$ peak in high-resolution XPS spectra of Se 3d orbitals (Figures S12 and S13). Furthermore, there is no indication of oxidized Cd 3d peaks in the high-resolution XPS spectra for all our doped and undoped samples stored over long time in ambient conditions.

Here among different series of doped-NPLs synthesized by the same partial CE method, we obtained the highest PL QY to be around $63 \%$. To achieve the dominant and efficient dopant emission quickly through these partial $\mathrm{CE}$ reactions, we investigated the effect of temperature. Surprisingly, annealing of the doped-NPL ensembles in the solution-state shows the transformation of doped-NPLs emitting both $\mathrm{BE}$ and $\mathrm{Cu}(\mathrm{I})$ emission to only $\mathrm{Cu}(\mathrm{I})$-related emission. Moreover, this shift results in an increase of the PL QY of doped samples. Figures 5a-5d depict UV-visible absorption and PL emission spectra for two samples before and after the annealing process. Consistently, both samples show annealing at $120^{\circ} \mathrm{C}$ under $\mathrm{N}_{2}$ for $30 \mathrm{~min}$, which leads to successful elimination of the $\mathrm{BE}$ emission and then the successful $\mathrm{Cu}(\mathrm{I})$ emission with very high PL QYs. We observed that after annealing the PL QY increases from $21 \%$ to $44 \%$ and $28 \%$ to $51 \%$ for the cases of acetate- and nitrate-based samples, respectively. Time-resolved fluorescence measurements before and after the annealing indicate that the PL decay curves slow down, suggesting the suppression of the competing nonradiative channels (see Figure S21 and Table S1). Moreover, conversion of doped NPLs emitting from the band-edge and dopant emission to only dominant dopant emission and redshift of dopant peak supports our earlier presented emission mechanism. Briefly, annealing of the samples synthesized using copper acetate precursor shows larger redshift $(692-764 \mathrm{~nm})$ as compared to doped NPLs synthesized by using nitrate precursor $(728-758 \mathrm{~nm})$. As discussed previously, the redshift in $\mathrm{Cu}$ emission is governed by increased substitutionally doped dopant ions which creates hybridized states below $\mathrm{CB}$ edge. ${ }^{22,40}$ Furthermore, after annealing, the percentage contribution of $\mathrm{Cu}$ emission increases from 88.2 and 99.4 to $\sim 100 \%$ for both acetate and nitrate-based samples, respectively. This shift in dopant emission contribution is consistent with the degree of redshift in dopant emission for both the cases. Although annealing helps to achieve full dopant emission, longer-time annealing at these temperatures is found to degrade the excitonic features of NPLs (Figure S22). Therefore, collective optimization of the experimental parameters with respect to the annealing temperature, time and environment may help to further increase the absolute PL QY of these doped-NPLs.

The spectral tunability of the $\mathrm{Cu}$-induced emission in these doped NPLs was also investigated through the synthesis of $\mathrm{Cu}$ doped NPLs having varying vertical thicknesses including 3 and 5 MLs via the versatile partial CE technique (see the experimental section in the Supporting Information). Figure 6 exhibits the absorption and PL emission spectra of all our doped NPLs. As seen from the figure, it is conveniently possible to tune the $\mathrm{Cu}(\mathrm{I})$-related emission in the doped NPL ensembles from 640 to $830 \mathrm{~nm}$.

Finally, temperature-dependent PL decay dynamics in the doped-NPL ensemble were also examined for the exemplary case of $1.7 \% \mathrm{Cu}$ doping. Figure $7 \mathrm{a}$ shows the PL decay curves in the temperature range of $30-270 \mathrm{~K}$. As the temperature was progressively decreased, we observed that the PL decay of the

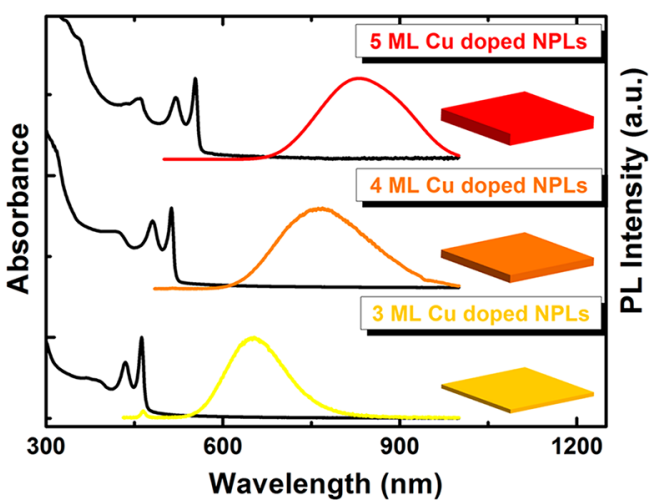

Figure 6. UV absorption and PL emission spectra of (a) $3 \mathrm{ML}$ (yellow), $4 \mathrm{ML}$ (orange), and $5 \mathrm{ML}$ (red) Cu-doped CdSe NPLs. The highest PL QYs for the 3-5 ML Cu-doped NPLs are 28, 63, and 51\%, respectively.

dopant emission slows down. As shown in Figure $7 \mathrm{~b}$, both amplitude-averaged lifetime and the longest lifetime component increase with the decreasing temperature toward cryogenic temperatures. Below $60 \mathrm{~K}$, a monotonic increase of the PL lifetime can be observed, which has previously been similarly reported for the $\mathrm{Cu}(\mathrm{I})$-doped $\mathrm{CdSe}$, InP, and $\mathrm{CuInS}_{2} \mathrm{CQDs}^{36}$ This temperature-dependent increase in the lifetime for these NCs has been recently attributed to a magnetic-exchange splitting within the luminescent excited state. ${ }^{36}$ A more detailed investigation of the temperature-dependent emission kinetics in these $\mathrm{Cu}(\mathrm{I})$ doped NPLs will also be of interest to the community to understand deeper into the photophysics of these materials.

\section{DISCUSSION}

The presented systematic and detailed studies of $\mathrm{Cu}(\mathrm{I})$ doping in the CdSe NPLs through the partial CE method provide a comprehensive understanding of the doping mechanism in these strongly confined quantum systems. First, the $1+$ oxidation state of $\mathrm{Cu}$-dopant ions in the host core-only $\mathrm{CdSe}$ NPLs as confirmed by our XPS experiments was corroborated along with optical studies. Second, the careful control of the partial CE reactions and annealing of the nonuniform doped samples have resulted in almost $100 \%$ dopant induced PL emission. As discussed in the literature for $\mathrm{Cu}(\mathrm{I})$-doped $\mathrm{CdSe}$ CQDs, $\mathrm{Cu}$ in $1+$ oxidation state fundamentally cannot show any $\mathrm{BE}$ recombination. The photoexcited holes in the host $\mathrm{CdSe}$ are captured by $\mathrm{Cu}(\mathrm{I})$ ions, which in turn recombine with delocalized electrons of CB leading to dominant Stokes-shifted dopant induced PL emission. Therefore, these atomically flat NPLs exhibit similar PL emission mechanism as in the case of $\mathrm{Cu}(\mathrm{I})$-doped CQDs. The only appearing difference is, due to the large lateral area of the NPLs, the requirement of further experimental procedures to be controllable for achieving full $\mathrm{Cu}$-based emission. Therefore, this study in general allows us to control and understand the evolution of dopant-related emission in strong $\mathrm{QC}$ regimes.

In the past decade, for these $\mathrm{Cu}$-doped CdSe CQDs, apart from their unique optical and electronic properties, there has been a tremendous interest in the scientific community for understanding chemical dopant-induced hybridization of the host CdSe orbitals. In the past, tuning the bandgap (changing both VB and $\mathrm{CB}$ ) with the size variation and alloying of CQDs was readily achievable. ${ }^{45,46}$ However, controlling the position of 

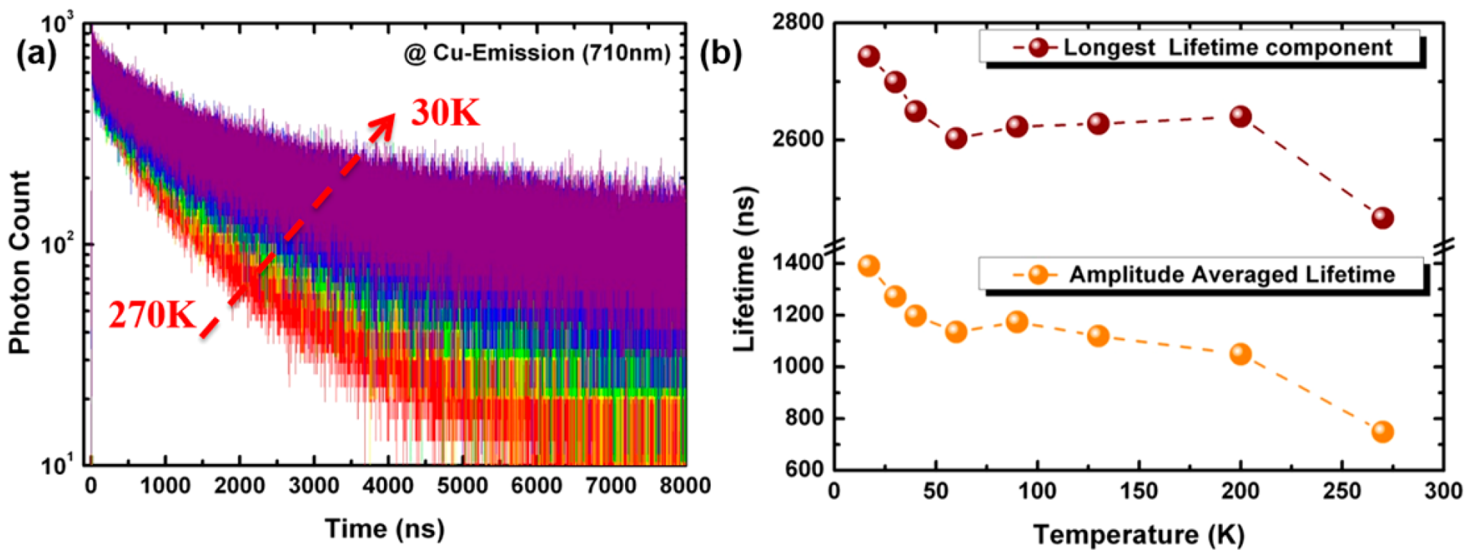

Figure 7. (a) The PL decay profile of Cu-emission in CdSe NPLs at different cryogenic temperatures (red @270 K and violet @30 K) and (b) amplitude-averaged and longest lifetimes for $\mathrm{Cu}$-doped NPLs as a function of the measurement temperature ranging from 270 to $17 \mathrm{~K}$.

individual $\mathrm{VB} / \mathrm{CB}$ edges of $\mathrm{CQDs}$ with respect to photocathode/anode is needed for their practical applications in future CQD based devices. ${ }^{22}$ Meulenberg et al. have shown in the past few years that increasing the amount of $\mathrm{Cu}(\mathrm{I})$-dopant ions in the host CdSe CQDs affects the lowest empty electronic states (CB) in a way inconsistent with the quantum confinement (QC) theory. ${ }^{40}$ Concisely, increasing $\mathrm{Cu}(\mathrm{I})$ dopant ions in CdSe CQDs results in the decrease of $\mathrm{CB}$ edge (only), which further shifts the PL emission spectrum to longer wavelengths. Meulenberg et al. have explained these results as a result of the hybridization of CdSe orbitals by successful substitution of $\mathrm{Cu}(\mathrm{I})$ ions in the CdSe host medium proved this hypothesis through theoretical modeling, X-ray absorption (XAS) spectroscopy ${ }^{40}$ and, more recently, ultraviolet photoemission spectroscopy (UPS). ${ }^{22}$ However, presence of these electronically and optically active states below the $\mathrm{CB}$ due to $\mathrm{Cu}(\mathrm{I})$ doping and redshifting of emission spectra by increasing the number of dopant ions in the host lattice can be confused with the particle size distribution inherent within the ensemble of doped CdSe CQDs. To this end, strongly confined quasi-2D NPLs may serve as better systems to test these interesting theoretical and experimental observations. Here, the excitonic structure is mostly invariable due to the lateral size of the NPLs and the emission is originating from vertically confined direction, which is fixed for these NPLs. Therefore, any increase in $\mathrm{Cu}(\mathrm{I})$ doping at substitutional sites in $\mathrm{CdSe}$ NPLs should result in a redshift of the emission spectrum (and vice versa) according to this hybridization theory. In our detailed partial CE experiments, the consistent redshift of PL emission spectra with increasing doping strongly supports the hybridization of CB edge states in the host CdSe NPLs. Figures 2c, 2f, 4d-4f, and Figures S14-S16 show the systematic variation of the dopant emission peak wavelength with the increasing incorporation of $\mathrm{Cu}(\mathrm{I})$ ions in NPLs. Additionally, use of the same core for doping with different amounts of $\mathrm{Cu}(\mathrm{I})$ via the partial $\mathrm{CE}$ method provides an outstanding set of samples for understanding chemical dopant induced orbital hybridization model. Furthermore, the appearance of $\mathrm{Cu}(\mathrm{I})$ related emission spectra with smaller energies than bandgap in excitation-dependent PL emission spectroscopy and the depth profile XPS results also confirm successful doping of $\mathrm{Cu}(\mathrm{I})$ in the host CdSe NPLs (see the Supporting Information, Figure S23, Table S2, and Sections E and F for a detailed discussion).

In conclusion, we have carried out an elaborative and systematic study on the $\mathrm{Cu}(\mathrm{I})$ doping process in strongly $1 \mathrm{D}$ - confined CdSe NPLs possessing exceptional optical properties including the tunable PL emission in the visible-to-NIR spectral region and large Stokes shift accompanied by high PL QYs and large absorption cross sections compared to doped CQDs. Here detailed XPS, steady-state PL, and temperature-dependent time-resolved fluorescence spectroscopy investigations verified the successful $\mathrm{Cu}(\mathrm{I})$ doping in CdSe NPLs via the partial CE method proposed and developed for NPLs for the first time in this study. In this work, the slow doping of $\mathrm{Cu}(\mathrm{I})$ in CdSe uniquely allowed to develop a deep understanding of the photophysics of dopant emission in strong $\mathrm{QC}$ regimes. The combination of stable and dominant dopant-related PL emission having minimum self-absorption along with signifcantly unchanging PL QYs at higher optical densities confirmed the advantages of successful doping of $\mathrm{Cu}(\mathrm{I})$ in CdSe NPLs. This simple yet powerful technique, together with the understanding of the doping process, enables the controlled $\mathrm{Cu}(\mathrm{I})$ doping in NPLs, which could be extended to different transition metal elements for the next-generation optoelectronic and color conversion devices.

\section{EXPERIMENTAL SECTION}

Synthesis of $4 \mathrm{ML}$ Thick CdSe Nanoplatelets. For a typical synthesis, $340 \mathrm{mg}$ of cadmium myristate, $24 \mathrm{mg}$ of Se, and $30 \mathrm{~mL}$ of ODE were loaded into a $100 \mathrm{~mL}$ three-neck flask. The solution was degassed and stirred at $95{ }^{\circ} \mathrm{C}$ under vacuum for an hour to evaporate volatile solvents and dissolve the cadmium myristate completely. Then, the heater was set to $240{ }^{\circ} \mathrm{C}$, the vacuum was broken at $100{ }^{\circ} \mathrm{C}$, and the flask was filled with argon gas. As the temperature reached 195 ${ }^{\circ} \mathrm{C}$, the color of the solution became yellowish, and at this stage 120 $\mathrm{mg}$ of cadmium acetate dihydrate was introduced swiftly into the reaction. After the growth of CdSe NPLs at $240{ }^{\circ} \mathrm{C}$ for around $10 \mathrm{~min}$, $1 \mathrm{~mL}$ of $\mathrm{OA}$ was injected, and the temperature of the solution was decreased to room temperature using a water bath. The solution was centrifuged for $5 \mathrm{~min}$ at $6000 \mathrm{rpm}$, and the supernatant was removed into another centrifuge tube. After adding ethanol into the supernatant solution until it became turbid, the solution was centrifuged again at $6000 \mathrm{rpm}$ for $10 \mathrm{~min}$, and then the precipitates were dissolved and stored in toluene. The synthesis of 3 and 5 ML CdSe core NPLs is given in the Supporting Information.

Partial Cation-Exchange. All exchange reactions were carried out in an oxygen- and water-free glovebox. CdSe NPL dispersion having $2.68 \times 10^{-6} \mathrm{~mol} / \mathrm{L}$ concentration was kept under stirring at $60^{\circ} \mathrm{C}$. For a typical partial cation-exchange reaction, $1 \mathrm{~mL}$ of the above CdSe NPL dispersion was diluted to $4 \mathrm{~mL}$ in toluene. The first dopant precursor was prepared by mixing $1 \mathrm{~mL}$ of $0.4 \mathrm{M}$ ethanolic solution of $\mathrm{Cu}(\mathrm{OAc})_{2}$ with $1.5 \mathrm{~mL}$ of TOP. It was kept under stirring for $30 \mathrm{~min}$ 
at $45{ }^{\circ} \mathrm{C}$. Similarly, the second dopant precursor is prepared by mixing $1 \mathrm{~mL}$ of $0.1 \mathrm{M}$ ethanolic solution of $\mathrm{Cu}\left(\mathrm{NO}_{3}\right)_{2}$ with $1.5 \mathrm{~mL}$ of TOP in $30 \mathrm{~min}$ at $45{ }^{\circ} \mathrm{C}$. It has been shown for QDs that TOP, being a soft base, binds to $\mathrm{Cu}$ (II) (intermediate soft acid), reducing the reactivity of dopant ions toward Se of CdSe NCs, which avoids full conversion of $\mathrm{CdSe}$ to $\mathrm{Cu}_{2} \mathrm{Se}$ phases. ${ }^{2,20}$ Ethanol present in the solution was thus used to extract replaced Cd(II) ions in the NPLs. Different amounts of dopant precursors were prepared to achieve different doping concentrations. However, the total volume of the cationic solution was kept the same for each reaction with the addition of a premixed solution of TOP (3 vol \%) and ethanol (2 vol \%) for maintaining a fixed concentration of TOP and ethanol in all reactions. Using 50,100, 150,200 , and $250 \mu \mathrm{L}$ of the stock solution with $1 \mathrm{~mL}$ of CdSe NPL dispersion $\left(6.7 \times 10^{-7} \mathrm{~mol} / \mathrm{L}\right)$ leads to $0.5,0.9,1.7,3.2$, and $3.6 \%$ $((\mathrm{Cu} / \mathrm{Cu}+\mathrm{Cd}) \%)$ doping of $\mathrm{Cu}(\mathrm{I})$ in $4 \mathrm{ML}$ CdSe NPLs. Similarly, 20, $40,60,80,100,120,140$, and $160 \mu \mathrm{L}$ of the stock solution of $\mathrm{Cu}\left(\mathrm{NO}_{3}\right)_{2}$ added in $1 \mathrm{~mL}$ of CdSe NPL dispersion $\left(6.7 \times 10^{-7} \mathrm{~mol} /\right.$ $\mathrm{L})$ resulted in $0.2,0.4,0.9,1.2,1.7,2.5,2.8$, and $3.2 \%((\mathrm{Cu} / \mathrm{Cu}$ $+\mathrm{Cd}) \%$ ) doping of $\mathrm{Cu}(\mathrm{I})$ in $4 \mathrm{ML}$ CdSe NPLs. A similar method was followed for doping of 3 and 5 ML CdSe NPLs.

After adding the dopant precursor, the samples were stirred vigorously, and the solution was allowed to equilibrate while stirring for $1 \mathrm{~h}$. After the completion of reaction, all the samples were precipitated with excess ethanol and washed several times using excess ethanol and dispersed in toluene and kept on stirring at $50{ }^{\circ} \mathrm{C}$. Absorption and photoluminescence spectra were measured at different time intervals (1-9 days) to study the effect of doping these thin NPLs.

TRF Spectroscopy. Time-resolved fluorescence (TRF) spectroscopy measurements were performed by using a time-correlated single photon-counting (TCSPC) system (PicoQuant FluoTime 200, PicoHarp 300). We used the picosecond pulsed laser (PicoQuant) at $375 \mathrm{~nm}$ for excitation, and the laser intensity was kept low $(\sim 1 \mathrm{~nJ} /$ $\left.\mathrm{cm}^{2}\right)$ so that the number of photogenerated excitons per NPL was much less than $1(\langle N\rangle \ll 1)$. In addition, the TRF spectroscopy measurements at cryogenic temperatures were carried out using a closed-cycle He cryostat that is coupled with our TRF spectroscopy system. The measurements at room temperature were conducted in solution form of NPL samples using a quartz cuvette, whereas the measurements at cryogenic temperatures were carried out in their solid film forms on Si substrates using drop-casted samples. To analyze the photoluminescence decay curves, the photoluminescence decay curves were fitted with multiexponential functions using FluoFit software in reconvolution mode.

In our low temperature TRF measurements, the pressure of the sample environment was $\sim 0.0045 \mathrm{mmHg}$, and, according to our calculations, the boiling point of TOP under this pressure is $\sim 283 \mathrm{~K}$. In addition, we intentionally increased the temperature up to $330 \mathrm{~K}$ under vacuum and then decreased it to cryogenic temperatures. Therefore, the possible inconsistency in the emission kinetics of the NPLs due to the differences in TOP amount was eliminated by allowing the complete evaporation of TOP under low pressure conditions. In light of this information, we could obtain the TRF decays without the interference of the effects of TOP and at low temperatures ranging from 270 to $17 \mathrm{~K}$, which is shown in Figure 7.

\section{ASSOCIATED CONTENT}

\section{S Supporting Information}

The Supporting Information is available free of charge on the ACS Publications website at DOI: 10.1021/acs.chemmater.8b00196.

Additional UV-visible absorption, steady-state excitation, and emission analysis, structural and elemental analyses which include additional HAADF based EDS images, XRD, HRTEM, and XPS analyses, additional results for partial $\mathrm{CE}$ experiments, excitation wavelength dependent PL emission studies, XPS depth profile studies, effect of using TOP in doped NPLs and control sample (undoped NPLs), additional materials and methods (PDF)

\section{AUTHOR INFORMATION}

\section{Corresponding Author}

*E-mail: volkan@stanfordalumni.org, hvdemir@ntu.edu.sg.

ORCID $\odot$

Burak Guzelturk: 0000-0003-1977-6485

James R. McBride: 0000-0003-0161-7283

Hilmi Volkan Demir: 0000-0003-1793-112X

\section{Notes}

The authors declare no competing financial interest.

\section{ACKNOWLEDGMENTS}

We gratefully acknowledge the financial support from the National Research Foundation of Singapore under its Investigatorship program (NRF-NRFI2016-08). H.V.D. also acknowledges TUBA.

\section{REFERENCES}

(1) Khan, A. H.; Dalui, A.; Mukherjee, S.; Segre, C. U.; Sarma, D. D.; Acharya, S. Efficient Solid-State Light-Emitting CuCdS Nanocrystals Synthesized in Air. Angew. Chem., Int. Ed. 2015, 54, 2643-2648.

(2) Sahu, A.; Kang, M. S.; Kompch, A.; Notthoff, C.; Wills, A. W.; Deng, D.; Winterer, M.; Frisbie, C. D.; Norris, D. J. Electronic Impurity Doping in CdSe Nanocrystals. Nano Lett. 2012, 12, 25872594.

(3) Yang, L.; Knowles, K. E.; Gopalan, A.; Hughes, K. E.; James, M. C.; Gamelin, D. R. One-Pot Synthesis of Monodisperse Colloidal Copper-Doped CdSe Nanocrystals Mediated by Ligand-Copper Interactions. Chem. Mater. 2016, 28, 7375-7384.

(4) Srivastava, B. B.; Jana, S.; Pradhan, N. Doping Cu in Semiconductor Nanocrystals: Some Old and Some New Physical Insights. J. Am. Chem. Soc. 2011, 133, 1007-1015.

(5) Stouwdam, J. W.; Janssen, R. A. J. Electroluminescent Cu-Doped CdS Quantum Dots. Adv. Mater. 2009, 21, 2916-2920.

(6) Viswanatha, R.; Brovelli, S.; Pandey, A.; Crooker, S. A.; Klimov, V. I. Copper-Doped Inverted Core/shell Nanocrystals With "permanent" optically Active Holes. Nano Lett. 2011, 11, 4753-4758.

(7) Wang, X.; Yan, X.; Li, W.; Sun, K. Doped Quantum Dots for White-Light-Emitting Diodes Without Reabsorption of Multiphase Phosphors. Adv. Mater. 2012, 24, 2742-2747.

(8) Yu, J. H.; Liu, X.; Kweon, K. E.; Joo, J.; Park, J.; Ko, K.-T.; Lee, D. W.; Shen, S.; Tivakornsasithorn, K.; Son, J. S.; et al. Giant Zeeman Splitting in Nucleation-Controlled Doped CdSe: $\mathrm{Mn}^{2+}$ Quantum Nanoribbons. Nat. Mater. 2010, 9, 47-53.

(9) Jawaid, A. M.; Chattopadhyay, S.; Wink, D. J.; Page, L. E.; Snee, P. T. Cluster-Seeded Synthesis of Doped CdSe:Cu $\mathrm{Cu}_{4}$ Quantum Dots. ACS Nano 2013, 7, 3190-3197.

(10) Meulenberg, R. W.; van Buuren, T.; Hanif, K. M.; Willey, T. M.; Strouse, G. F.; Terminello, L. J. Structure and Composition of $\mathrm{Cu}-$ Doped CdSe Nanocrystals Using Soft X-Ray Absorption Spectroscopy. Nano Lett. 2004, 4, 2277-2285.

(11) Li, Z.; Peng, X. Size/shape-Controlled Synthesis of Colloidal CdSe Quantum Disks: Ligand and Temperature Effects. J. Am. Chem. Soc. 2011, 133, 6578-6586.

(12) Chen, D.; Gao, Y.; Chen, Y.; Ren, Y.; Peng, X. Structure Identification of Two-Dimensional Colloidal Semiconductor Nanocrystals with Atomic Flat Basal Planes. Nano Lett. 2015, 15, 44774482.

(13) Thoma, S. G.; Sanchez, A.; Provencio, P. P.; Abrams, B. L.; Wilcoxon, J. P. Synthesis, Optical Properties, and Growth Mechanism of Blue-Emitting CdSe Nanorods. J. Am. Chem. Soc. 2005, 127, 76117614. 
(14) Boldt, K.; Kirkwood, N.; Beane, G. A.; Mulvaney, P. Synthesis of Highly Luminescent and Photo-Stable, Graded Shell CdSe $/ \mathrm{Cd}_{\mathrm{x}} \mathrm{Zn}_{1-\mathrm{x}} \mathrm{S}$ Nanoparticles by in Situ Alloying. Chem. Mater. 2013, 25, 4731-4738.

(15) Saunders, A. E., Shieh, F. General Shape Control of Colloidal Cds, Cdse and Cdte Semiconductor Nanorods and Nanorod Heterostructures. AIChE Annual Meeting, Conference Proceedings 2005, 4640.

(16) White, S. L.; Smith, J. G.; Behl, M.; Jain, P. K. Co-Operativity in a Nanocrystalline Solid-State Transition. Nat. Commun. 2013, 4, 2933.

(17) Beberwyck, B. J.; Surendranath, Y.; Alivisatos, A. P. Cation Exchange: A Versatile Tool for Nanomaterials Synthesis. J. Phys. Chem. C 2013, 117, 19759-19770.

(18) Lesnyak, V.; Brescia, R.; Messina, G. C.; Manna, L. Cu Vacancies Boost Cation Exchange Reactions in Copper Selenide Nanocrystals. J. Am. Chem. Soc. 2015, 137, 9315-9323.

(19) Bouet, C.; Laufer, D.; Mahler, B.; Nadal, B.; Heuclin, H.; Pedetti, S.; Patriarche, G.; Dubertret, B. Synthesis of Zinc and Lead Chalcogenide Core and Core/shell Nanoplatelets Using Sequential Cation Exchange Reactions. Chem. Mater. 2014, 26, 3002-3008.

(20) Gopal, M. B. Ag and Cu Doped Colloidal CdSe Nanocrystals: Partial Cation Exchange and Luminescence. Mater. Res. Express 2015, 2, 085004 .

(21) De Trizio, L.; Prato, M.; Genovese, A.; Casu, A.; Povia, M.; Simonutti, R.; Alcocer, M. J. P.; D’Andrea, C.; Tassone, F.; Manna, L. Strongly Fluorescent Quaternary Cu-In-Zn-S Nanocrystals Prepared from $\mathrm{Cu}_{1-\mathrm{x}} \mathrm{InS}_{2}$ Nanocrystals by Partial Cation Exchange. Chem. Mater. 2012, 24, 2400-2406.

(22) Wright, J. T.; Forsythe, K.; Hutchins, J.; Meulenberg, R. W. Implications of Orbital Hybridization on the Electronic Properties of Doped Quantum Dots: The Case of Cu:CdSe. Nanoscale 2016, 8, 9417-9424.

(23) Ithurria, S.; Dubertret, B. Quasi 2D Colloidal CdSe Platelets with Thicknesses Controlled at the Atomic Level. J. Am. Chem. Soc. 2008, 130, 16504-16505.

(24) Ithurria, S.; Tessier, M. D.; Mahler, B.; Lobo, R. P. S. M.; Dubertret, B.; Efros, A. L. Colloidal Nanoplatelets with TwoDimensional Electronic Structure. Nat. Mater. 2011, 10, 936-941.

(25) Kelestemur, Y.; Guzelturk, B.; Erdem, O.; Olutas, M.; Gungor, K.; Demir, H. V. Platelet-in-Box Colloidal Quantum Wells: CdSe/ CdS@CdS Core/Crown@Shell Heteronanoplatelets. Adv. Funct. Mater. 2016, 26, 3570-3579.

(26) Olutas, M.; Guzelturk, B.; Kelestemur, Y.; Yeltik, A.; Delikanli, S.; Demir, H. V. Lateral Size-Dependent Spontaneous and Stimulated Emission Properties in Colloidal CdSe Nanoplatelets. ACS Nano 2015, 9, 5041-5050.

(27) Yeltik, A.; Delikanli, S.; Olutas, M.; Kelestemur, Y.; Guzelturk, B.; Demir, H. V. Experimental Determination of the Absorption Cross-Section and Molar Extinction Coefficient of Colloidal CdSe Nanoplatelets. J. Phys. Chem. C 2015, 119, 26768-26775.

(28) Guzelturk, B.; Kelestemur, Y.; Olutas, M.; Delikanli, S.; Demir, H. V. Amplified Spontaneous Emission and Lasing in Colloidal Nanoplatelets. ACS Nano 2014, 8, 6599-6605.

(29) She, C.; Fedin, I.; Dolzhnikov, D. S.; Dahlberg, P. D.; Engel, G. S.; Schaller, R. D.; Talapin, D. V. Red, Yellow, Green, and Blue Amplified Spontaneous Emission and Lasing Using Colloidal CdSe Nanoplatelets. ACS Nano 2015, 9, 9475-9485.

(30) Wang, Y.; Zhukovskyi, M.; Tongying, P.; Tian, Y.; Kuno, M. Synthesis of Ultrathin and Thickness-Controlled $\mathrm{Cu}_{2-\mathrm{x}} \mathrm{Se}$ Nanosheets via Cation Exchange. J. Phys. Chem. Lett. 2014, 5, 3608-3613.

(31) Izquierdo, E.; Robin, A.; Keuleyan, S.; Lequeux, N.; Lhuillier, E.; Ithurria, S. Strongly Confined HgTe 2D Nanoplatelets as Narrow Near-Infrared Emitters. J. Am. Chem. Soc. 2016, 138, 10496-10501.

(32) Sharma, M.; Gungor, K.; Yeltik, A.; Olutas, M.; Guzelturk, B.; Kelestemur, Y.; Erdem, T.; Delikanli, S.; McBride, J. R.; Demir, H. V. Near-Unity Emitting Copper-Doped Colloidal Semiconductor Quantum Wells for Luminescent Solar Concentrators. Adv. Mater. 2017, 29, 1700821

(33) Knowles, K. E.; Hartstein, K. H.; Kilburn, T. B.; Marchioro, A.; Nelson, H. D.; Whitham, P. J.; Gamelin, D. R. Luminescent Colloidal
Semiconductor Nanocrystals Containing Copper: Synthesis, Photophysics, and Applications. Chem. Rev. 2016, 116, 10820-10851.

(34) Whitham, P. J.; Knowles, K. E.; Reid, P. J.; Gamelin, D. R. Photoluminescence Blinking and Reversible Electron Trapping in Copper-Doped CdSe Nanocrystals. Nano Lett. 2015, 15, 4045-4051.

(35) Grandhi, G. K.; Viswanatha, R. Tunable Infrared Phosphors Using Cu Doping in Semiconductor Nanocrystals: Surface Electronic Structure Evaluation. J. Phys. Chem. Lett. 2013, 4, 409-415.

(36) Knowles, K. E.; Nelson, H. D.; Kilburn, T. B.; Gamelin, D. R. Singlet-Triplet Splittings in the Luminescent Excited States of Colloidal $\mathrm{Cu}^{+}: \mathrm{CdSe}, \mathrm{Cu}^{+}: \mathrm{InP}$, and $\mathrm{CuInS}_{2}$ Nanocrystals: ChargeTransfer Configurations and Self-Trapped Excitons. J. Am. Chem. Soc. 2015, 137, 13138-13147.

(37) Tang, A.; Yi, L.; Han, W.; Teng, F.; Wang, Y.; Hou, Y.; Gao, M. Synthesis, Optical Properties, and Superlattice Structure of $\mathrm{Cu}(\mathrm{I})$ Doped CdS Nanocrystals. Appl. Phys. Lett. 2010, 97, 033112.

(38) Pandey, A.; Brovelli, S.; Viswanatha, R.; Li, L.; Pietryga, J. M.; Klimov, V. I.; Crooker, S. A. Long-Lived Photoinduced Magnetization in Copper-Doped $\mathrm{ZnSe}-\mathrm{CdSe}$ Core-shell Nanocrystals. Nat. Nanotechnol. 2012, 7, 792-797.

(39) Hazarika, A.; Layek, A.; De, S.; Nag, A.; Debnath, S.; Mahadevan, P.; Chowdhury, A.; Sarma, D. D. Ultranarrow and Widely Tunable $\mathrm{Mn}^{2+}$-Induced Photoluminescence from Single $\mathrm{Mn}$ Doped Nanocrystals of ZnS-CdS Alloys. Phys. Rev. Lett. 2013, 110, 267401.

(40) Wright, J. T.; Meulenberg, R. W. Effects of Dopants on the Band Structure of Quantum Dots: A Theoretical and Experimental Study. Phys. Rev. B: Condens. Matter Mater. Phys. 2013, 88, 45432.

(41) Nelson, H. D.; Li, X.; Gamelin, D. R. Computational Studies of the Electronic Structure $\mathrm{S}$ of Copper-Doped CdSe Nanocrystals: Oxidation States, Jahn-Teller Distortions, Vibronic Bandshapes, and Singlet-Triplet Splittings. J. Phys. Chem. C 2016, 120, 5714-5723.

(42) Radi, A.; Pradhan, D.; Sohn, Y.; Leung, K. T. Nanoscale Shape and Size Control of Cubic, Cuboctahedral, and Octahedral $\mathrm{Cu}-\mathrm{Cu}_{2} \mathrm{O}$ Core-Shell Nanoparticles on $\mathrm{Si}(100)$ by One-Step, Templateless, Capping-Agent-Free Electrodeposition. ACS Nano 2010, 4, 15531560.

(43) Mahler, B.; Guillemot, L.; Bossard-Giannesini, L.; Ithurria, S.; Pierucci, D.; Ouerghi, A.; Patriarche, G.; Benbalagh, R.; Lacaze, E.; Rochet, F.; et al. Metallic Functionalization of CdSe 2D Nanoplatelets and Its Impact on Electronic Transport. J. Phys. Chem. C 2016, 120, 12351-12361.

(44) Katari, J. E. B.; Colvin, V. L.; Alivisatos, A. P. X-Ray Photoelectron Spectroscopy of CdSe Nanocrystals with Applications to Studies of the Nanocrystal Surface. J. Phys. Chem. 1994, 98, 41094117.

(45) Murray, C. B.; Norris, D. J.; Bawendi, M. G. Synthesis and Characterization of Nearly Monodisperse CdE $(\mathrm{E}=\mathrm{S}$, Se, Te $)$ Semiconductor Nanocrystallites. J. Am. Chem. Soc. 1993, 115, 87068715.

(46) Bailey, R. E.; Nie, S. Alloyed Semiconductor Quantum Dots: Tuning the Optical Properties without Changing the Particle Size. J. Am. Chem. Soc. 2003, 125, 7100-7106. 